\title{
Central Zone of Left Half Prostate
}

National Cancer Institute

\section{Source}

National Cancer Institute. Central Zone of Left Half Prostate. NCI Thesaurus. Code

C128587.

The portion of the central zone of the prostate that is located to the anatomical left of center. 